Revue d'histoire de l'Amérique française

REVUE D'HISTOIRE DE L'AMÉRIQUE FRANÇAISE

\title{
L’Amérique du Nord et la Sacrée Congrégation « de Propaganda
} Fide ", 1622-1799

\section{Guides et Inventaires}

\section{Luca Codignola}

Volume 33, numéro 2, septembre 1979

URI : https://id.erudit.org/iderudit/303775ar

DOI : https://doi.org/10.7202/303775ar

Aller au sommaire du numéro

Éditeur(s)

Institut d'histoire de l'Amérique française

ISSN

0035-2357 (imprimé)

1492-1383 (numérique)

Découvrir la revue

Citer cet article

Codignola, L. (1979). L’Amérique du Nord et la Sacrée Congrégation « de Propaganda Fide », 1622-1799 : guides et Inventaires. Revue d'histoire de l'Amérique française, 33(2), 197-214. https://doi.org/10.7202/303775ar d'utilisation que vous pouvez consulter en ligne. 


\section{L'AMÉRIQUE DU NORD ET LA SACRÉE CONGRÉGATION \\ "de Propaganda Fide", 1622-1799 \\ Guides et Inventaires*}

LUCA CODIGNOLA

Università di Pisa, Italie

Les historiens reconnaissent l'importance de l'Église catholique dans l'histoire de l'Amérique du Nord, et dans celle du Canada en particulier. Ils admettent aussi que les archives religieuses romaines renferment une documentation considérable, soit qu'elles concernent indirectement l'histoire nord-américaine (impressions et récits de voyageurs, par exemple), soit surtout qu'elles aient exercé sur elle une influence immédiate (directives aux missionnaires, facultés concédées aux évêques, positions prises devant certains événements ou attitude adoptée à l'égard des Indiens, par exemple). Pourtant, comme le note David Beers Quinn, en faisant le décompte des sources de l'histoire coloniale nord-américaine qui sont conservées dans les dépôts d'archives européens, ce sont ces mêmes archives romaines qu'on connaît le moins ${ }^{1}$.

Les archives de la Sacrée Congrégation "de Propaganda Fide" sont sans doute les plus importantes de toutes les archives romaines, "more important than all others taken together", écrivait dès 1911 Carl Russell Fish ${ }^{2}$. Si, en effet, la juridiction de la Propagande sur les colonies de l'Amérique britannique du Nord ne fut clairement définie que dans la deuxième moitié du XVIIIe siècle, le Canada

* Version revue d'une communication présentée au Terzo Convegno di Studi Canadesi (Urbino, Italie, 30 mai - ler avril 1979). La traduction française a été mise au point avec le concours de $M$. André Vachon, s.r.c.

1 David Beers Quinn, “Documenting Canada's Early White History”, Archivaria, 7 (Winter 1978): 86-94; voir, en particulier, p. 90. Quinn traite surtout du Canada, au XVIe siècle; vu le sens de l'article, j'ai cru pouvoir étendre la portée des affirmations de son auteur à toute l'Amérique du Nord, de même qu'à tout le XVIle siècle et à la première moitié du XVIIIe.

2 Fish, Guide, 121-122 (voir note 34 ).

[197]

RHAF, vol. 33, no 2, septembre 1979 
dépendit directement de cette congrégation dès 1622 et, sauf pendant les années 1674 à 1763, il resta sous sa juridiction jusqu'en 1908 , année où il passa sous le régime de droit commun ${ }^{3}$. Ce n'est donc pas par hasard que les États-Unis, grâce à l'initiative de l'Academy of Franciscan History ${ }^{4}$, et le Canada, grâce à la collaboration des Archives publiques du Canada et du Centre de Recherches en histoire religieuse du Canada, ont choisi de commencer leurs enquêtes sur les archives religieuses italiennes par celles de la Propagande.

Entrepris en 1955, le projet américain est en bonne voie de réalisation; le projet canadien fut annoncé et brièvement présenté par les Archives publiques du Canada dans L'Archiviste, à la fin d'octobre 19775. Les Canadiens entendent faire l'inventaire complet de tous les documents d'intérêt canadien conservés dans les Archives de la Propagande, depuis la création de cette congrégation en 1622 jusqu'à la date ultime d'accessibilité aux documents ${ }^{6}$, et non seulement pour tous les territoires actuels du Canada, même entrés récemment dans la Confédération, comme Terre-Neuve, mais aussi pour d'autres territoires qui firent partie de la Nouvelle-France, comme la Louisiane. Tout ce matériel documentaire sera en même temps microfilmé.

3 C'est Grégoire XV qui établit officiellement la compétence et les pouvoirs de la Propagande, le 22 juin 1622 (constitution Inscrutabili divinae Providentiae). Cette congrégation avait une double tâche: répandre la religion catholique chez les infidèles et protéger la foi dans les pays où les catholiques se trouvaient en contact avec des hérétiques ou des infidèles. Le 29 juin 1908 (constitution Sapienti consilio), Pie X exclut de la juridiction de la Propagande les Etats-Unis, le Canada, Terre-Neuve, l'Angleterre, l'Écosse, la Hollande et le Luxembourg (V. Niccolo Del Re, La curia romana. Lineamenti storico-giuridici, Rome, Edizioni di Storia e Letteratura, 1970): 185-203. Pour ce qui est du Canada, les années 1674 et 1763 marquent respectivement la création de l'évêché de Québec (confié à François de Laval, jusque-là vicaire apostolique du Canada) et la nomination au même évêché de Jean-Olivier Briand, premier évêque sous le Régime anglais. Ces limites chronologiques ne doivent toutefois pas être interprétées d'une façon trop rigoureuse, car il n'y eut ni cessation soudaine ni reprise immédiate des relations entre le Canada et la Propagande.

4 Voir, en particulier, la note 53.

5 Victorin Chabot, “Documents d'intérêt canadien à Rome", L'Archiviste, IV, 6 (novembre-décembre 1977): 3-4. Chabot est actuellement chef de la Section des Archives françaises, Division des Manuscrits, aux Archives Publiques du Canada.

6 Dans l'article, on affirme que la date ultime d'accessibilité aux archives de la Propagande, comme aux autres archives du Saint-Siège, est 1878. Or, depuis le 23 décembre 1978, il est possible de consulter tout le matériel archivistique jusqu'à l'année 1903. 
L'extension des frontières, dans le projet canadien, présente un aspect méthodologique important et rejoint cette remarque de Quinn, qu'il est temps de mettre en évidence "the early common roots of all the European settlements there, (...) exposing their common and comparable methods of exploring the far from empty land of the new continent", pour en arriver à voir "the early history of white activity in North America as a whole" effet, qu'établir entre les deux pays de l'Amérique du Nord une distinction trop nette, fondée sur les frontières politiques actuelles, serait une erreur. De vastes territoires qui font aujourd'hui partie des États-Unis: l'Arkansas, le Colorado, le Texas, l'Arizona, New Mexico, la Californie, la Floride, appartinrent d'abord à la Nouvelle-Espagne et ne devinrent "américains" qu'au cours du XIXe siècle. De même, une bonne partie de la Nouvelle-France: la Louisiane, l'Illinois, le Wisconsin, le Michigan, est maintenant comprise dans les Etats-Unis. Vu les objectifs de l'inventaire en cours, retenir Montréal et exclure Kaskakia n'aurait eu aucun sens; parallèlement, on ne comprendrait pas que les États-Unis retiennent Alburquerque et excluent Veracruz. Si donc, dans une perspective "canadienne", on peut choisir de ne pas s'intéresser à l'Amérique espagnole (laquelle eut avec l'Amérique française des rapports très limités, sauf en Louisiane), on ne peut absolument pas négliger la documentation relative à l'A mérique anglaise. Au demeurant - et ce point est fondamental - les fontionnairres romains eux-mêmes, y compris ceux de la Propagande, mirent passablement de temps à distinguer nettement les diverses unités territoriales du continent américain: la confusion entre l'Amérique du Nord, l'Amérique centrale et l'Amérique du Sud ne commencera à disparaître que bien après le début du XVIIIe siècle ${ }^{8}$. C'est pourquoi, dans la présente étude, on tient compte des différences - de juridiction, par exemple, - entre l'Amérique française et l'Amérique anglaise, mais on ne les dissocie pas. à la note 1 .

Quinn, “Documenting Canada's Early White History”, 93. Voir la remarque faite

8 Je ne veux pas dire qu'il n'existait pas de juridictions différentes ni de territoires bien définis, mais que, dans l'esprit des fonctionnaires romains (et ceux de la Propagande ne faisaient pas exception) les choses n'étaient pas très claires, et qu'on avait souvent tendance à confondre les pays. Cela est d'autant plus vrai que l'on remonte davantage dans le temps. 


\section{Guides et instruments de recherche}

L'oeuvre fondamentale de Fish ${ }^{9}$ mise à part, la première description des archives de la Propagande qui fasse référence à l'Amérique du Nord est celle de Peter K. Guilday ${ }^{10}$, lequel s'étonnait, en 1921, que cette documentation fût si peu connue des historiens nord-américains. C'est dans un article qu'on lui avait demandé à l'occasion du tricentenaire de la Propagande que Guilday trace brièvement l'histoire de cette congrégation, offrant au lecteur quelques indications bibliographiques générales sur la Curie romaine et sur la Propagande en particulier. Mais sa présentation des fonds d'archives, à peine ébauchée, est nettement insuffisante. Son article n'apportait rien de particulièrement nouveau, après ce qu'avait écrit Fish.

En revanche, l'essai de Conrad Morin, publié dans Culture ${ }^{11}$ au lendemain de la dernière guerre, est d'un grand intérêt. C'est une description approfondie des archives de la Propagande, conçue en fonction des études canadiennes mais comportant des points de référence utiles pour l'histoire des États-Unis. Après avoir brièvement décrit l'état des archives romaines et rappelé les premiers efforts du gouvernement canadien en vue de leur utilisation, Morin mentionne l'inventaire de Fish et l'oeuvre de Candide de Nant ${ }^{12}$, tout en notant que, par suite de la découverte de nouveaux documents et d'un reclassement partiel des fonds de la Propagande, ces travaux doivent être consultés avec une certaine prudence. Cet avertissement, incidemment, vaut non seulement pour Fish et de Nant, mais pour tous les chercheurs qui ont utilisé les archives de la Propagande avant les années trente. La partie centrale de l'essai de Morin est consacrée à la description des fonds de la Propagande ${ }^{13}$ d'abord, puis de ceux des Archives secrètes du Vatican ${ }^{14}$; cette partie comporte de nombreuses mentions de documents ou de registres dont le contenu intéresse le Canada. Dans sa description des fonds de la Propagande, Morin a préféré "le point de vue historique" au

\footnotetext{
9 Fish, Guide (V. note 34).

10 Peter K. Guilday, "The Sacred Congregation de Propaganda Fide (1622-1922)", The Catholic Historical Review, VI, 4 (January, 1921): 478-494.

"Conrad Morin, "Les archives du Saint-Siège, importantes sources de l'histoire politico-religieuse du Canada", Culture, VII, 2 (juin 1946): 151-176.

12 Candide de Nant, Pages glorieuses de l'épopée canadienne. Une mission Capucine en Acadie (Gembloux, Imprimerie J. Duculot, 1927).

13 Morin, "Archives du Saint-Siège": 156-161.

14 Ibid., 161-170.
} 
"point de vue archivistique"15; un autre ordre eût peut-être été plus utile au chercheur, obligé, pour retracer les documents, de convertir le classement historique de Morin en classement archivistique. Il faudrait en somme un deuxième inventaire (proprement archivistique, celui-là), qui permît d'utiliser celui de Morin. La dernière partie de l'essai porte sur les "archives canadiennes de contenu romain" et principalement sur les dépôts qui, au Canada, possèdent des "copies d'originaux" de Romel6.

On trouve aussi une description des archives de la Propagande dans un ouvrage collectif consacré aux sources de l'histoire de l'Amérique latine ${ }^{17}$. Par Amérique latine, il faut entendre ici non seulement les pays américains de langue espagnole ou portugaise, mais aussi, entre autres, d'anciens territoires français qui font maintenant partie des États-Unis, pendant la période précédant leur entrée dans la fédération ${ }^{18}$. Cet ouvrage contient donc une documentation intéressante pour le Canada et les États-Unis. Rédigé par Nicola Kowalsky, archiviste général de la Propagande de 1958 à 1966 , cet article, après une brève description des rapports de la Propagande avec l'Ámérique latine, présente une liste des différents fonds d'archives, accompagnée d'indications, certaines ou probables, des endroits où trouver des documents se rapportant aux territoires couverts par le volume. (À la différence de Morin, Kowalsky adopte une démarche archivistique.) Tout cet article est très sommaire, comme du reste la bibliographie qui en complète chaque partie, mais les éditeurs de l'ouvrage l'avaient ainsi voulu. Sans aucun doute, cet article reste néanmoins un utile instrument de travail.

Le dernier guide publié à ce jour est l'oeuvre de René Bélanger ${ }^{19}$. On y trouve une description de la Propagande, son

15 Morin affirme: "c'est le point de vue historique et non le point de vue archivistique (ou topographique) qui a inspiré le classement ci-dessous; en d'autres termes, dans le but de faciliter les recherches méthodiques, les collections ont été groupées par ordre d'appartenance de matières et non pas de fonds" (ibid., 155-156).

16 Ibid., 170-176. Sur la question des copies des documents de la Propagande conservées dans les archives et les bibliothèques de l'Amérique du Nord, voir dans la présente étude la partie consacrée aux "Documents de la Propagande en Amérique du Nord".

17 Nicola Kowalsky. "Archivio della Congregazione de Propaganda Fide", in Lajos Pásztor, éd., Guida delle fonti per la storia dell' America latina negli archivi della Santa Sede e negli archivi ecclesiastici d'Italia (Città del Vaticano, Archivio Vaticano, 1970): 328-338. 18 Ibid., vi.

19 René Bélanger, "Les archives historiques de la Propagande à Rome", Archives, 74, 1 (1974): 8-14. 
histoire et son fonctionnement, et une présentation assez détaillée des principales séries qui en composent les archives. C'est à la série Congressi que Bélanger s'attarde le plus, en indiquant certains documents d'intérêt canadien pour les années 1861 et 1862 . Un historique des rapports entre la Propagande et le Canada, et une bibliographie sommaire complètent ce court article qui, même s'ilest presque exclusivement tourné vers le XIXe siècle, complète utilement les guides de Morin et de Kowalsky.

Kowalsky a consacré une bonne partie de sa production scientifique aux archives de la Propagande. On lui doit un inventaire général de ces archives, qui est une oeuvre de premier ordre, unique, et absolument indispensable ${ }^{20}$. Après une courte introduction historique, Kowalsky passe en revue, série par série, tous les fonds qui constituent ces archives. Il indique brièvement la signification de chaque fonds dans l'histoire de la Propagande, et note, s'il y a lieu, les titres qui apparaissent au dos des volumes, ou encore le sujet de chacun. Certes, l'inventaire n'est exempt ni d'erreurs ni d'omissions: on peut en juger par les corrections et ajouts nombreux que les chercheurs ont portés dans les marges de l'exemplaire qui est à leur disposition à la salle de lecture des archives. Mais ces faiblesses, qui ne diminuent pas la valeur de ce précieux outil, ne pourront être éliminées qu'avec les années, au fur et à mesure que progresseront les recherches. On peut encore signaler un article de Kowalsky. "L'archivio della Sacra Congregazione 'de Propaganda Fide' "21, qu'on pourra lire, non sans profit, dans l'inventaire publié par Pasztor ${ }^{22}$; Kowalsky reprit aussi l'histoire des archives de la Propagande et de leurs archivistes ${ }^{23}$, et publia la liste annotée des préfets et des secrétaires généraux de cette congrégation ${ }^{24}$. Mais ce dernier ouvrage est maintenant dépassé par celui, plus récent, qu'a préparé l'actuel archiviste de la Propagande, Josef Metzler, lequel a compilé la liste des préfets de l'Économie et

20 Kowalsky, "Inventario dell'Archivo (sic) storico della S. Congregazione 'de Propaganda Fide", Neuen Zeitschrift für Missionswissenschaft, XVII (1961), 1: 9-23; 2: 109117; 3: 191-200.

21 Kowalsky, "L'archivio della Sacra Congregazione 'de Propaganda Fide' ", Rassegna degli Archivi di Stato, XXV, 1 gennaio-aprile 1965): 37-44.

22 Pasztor, éd., Guida delle fonti per la storia dell'Americana latina... (V. note 17).

23 Kowalsky, "L'archivio della Sacra Congregazione 'de Propaganda Fide' e i suoi archivisti”, Pontificia Universitas Gregoriana, Annales (1963-64): 38-53.

${ }_{24}$ Kowalsky, Serie dei cardinali prefetti e dei segretari della Sacra Congregazione “de Propaganda Fide" (Rome, Editiones Urbanianae, 1962). 
des secrétaires pour les Affaires orientales, en plus de celle des préfets généraux et des secrétaires généraux ${ }^{25}$. Ā Metzler encore, on doit un article fort utile, consacré aux index des archives de la Propagande ${ }^{26}$, entrepris dès 1657 , revus et augmentés par la suite.

\section{Inventaires}

Au chapitre des inventaires spécifiquement consacrés à l'Amérique du Nord, c'est en 1910 qu'est publié un premier inventaire 27 , anonyme, tiré de Fish ${ }^{28}$, et qui, selon son auteur, porterait sur le Canada, à l'exclusion des États-Unis, du Mexique et des Antilles. À vrai dire, la seule utilité de cette liste, dont la publication devance celle de Fish, est d'attirer l'attention sur l'importance des archives romaines. C'est une énumération de documents, répartis en séries (Acta, SOCG, Congressi, Memoriali, Scripta Varia), pour lesquels on fournit seulement la date (souvent présumée) et un sommaire d'une ligne, sans autre indication utile, - pas même la numérotation des folios. L'exclusion des Memoriali et des Scripta Varia ${ }^{29}$ des fonds de la Propagande révèle combien l'auteur était peu familier avec les documents dont il parle. Notons que, s'il a été question de faire faire des copies de ces documents pour les Archives canadiennes ${ }^{30}$, il ne semble pas que ce projet ait jamais été réalisé, quoi qu'en dise Morin ${ }^{31}$.

La partie de l'inventaire d'Ivanhoë Caron ${ }^{32}$, dressé de 1939 à 1942, qui concerne la Propagande est tirée du précédent. Caron compile, par ordre chronologique (de 1610 à 1729), une liste de

25 Josef Metzler, “Serie dei Cardinali Prefetti e dei Segretari della Sacra Congregazione", in Metzler, éd., Sacrae Congregationis de Propaganda Fide Memoria Rerum III/2 (Fribourg, Heider, 1976): 615-626.

26 Metzler, "Indici dell'archivo Storico della S.C. 'de Propaganda Fide' ”, Euntes Docete. Commentaria Urbaniana de Archivis et Bibliothecis Missionibus atque Scientiae Missionum Inservientibus, XXI (1968): 109-130.

${ }_{27}$ "Documents relatifs au Canada parmi les archives italiennes", Rapport de l'archiviste en chef du Canada (1910): 106-115. On y trouve la liste et une brève description des documents de la Bibliothèque Vaticane, de la Propagande et des Archives secrètes du Vatican.

28 Fish, Guide (V. note 34).

29 Ces deux séries n'existent plus sous ce titre. Mais Fish insère les Memoriali (pp. 189-190) et les Scripta Varia (p. 195) dans la section consacrée à la Propagande.

30 "Documents relatifs au Canada": 106.

31 Morin, "Archives du Saint-Siège": 154 (V. note 16).

32 Ivanhoë Caron, "Inventaire des documents concernant l'Église du Canada sous le régime français", Rapport de l'Archiviste de la Province de Québec (1939-40): 155-353; (194041): 333-473; (1941-42): 178-298. 
documents conservés dans différents dépôts d'archives, et aussi d'imprimés, qui ont trait à l'Église canadienne sous le Régime français. On n'y trouve aucune allusion à la Propagande, tout le matériel romain étant désigné comme "Documents du Vatican". De l'inventaire de 1910, Caron extrait tout ce qui lui paraît se rapporter à l'objet de ses recherches et l'intègre, chronologiquement, à sa compilation: opération de troisième main qui ne peut rien apporter de neuf sur la Propagande et ses archives. Dans son introduction ${ }^{33}$, Caron affirme que des copies des documents qu'il résume se trouvent tant aux Archives publiques du Canada qu'aux Archives de l'Archevêché de Québec.

Mais il est temps de parler, avec une insistance particulière, de l'ouvrage de Fish, Guide to the Materials for American History $y^{34}$. À la fois guide et inventaire, cité par tous les chercheurs, cet instrument de recherche fut, jusqu'à récemment, sans égal pour l'histoire de l'Amérique du Nord, et, à bien des égards, il n'a pas été dépassé. Fish a travaillé en Italie de septembre 1908 au début d'août 190935 , visitant un grand nombre de dépôts d'archives et de bibliothèques, qu'il décrit dans son ouvrage ${ }^{36}$, et accordant une attention particulière aux archives de la Propagande ${ }^{37}$. Malgré le titre de son inventaire, Fish examine "All general references to American or the Western Indies and all documents of interest for general American history (...) together with everything relating to Cuba and the northern provinces and bishoprics of Mexico, because of their connection with the missions of Florida and the Western States". ${ }^{38}$ De même, Fish s'est-il donné pour mission de faire connaitre "the unprinted sources for the history of the United States and Canada existing in Italy, and particularly at Rome". ${ }^{39}$

33 Caron précise: "Une autre source de renseignements (...) est indiquée dans le Rapport de l'archiviste en chef du Canada, pour l'année 1910 (p. 106), sous le titre 'Documents relatifs au Canada dans les archives italiennes'. Nous ne donnons qu'un sommaire de ces pièces écrites en langue italienne, mais le département des Archives Publiques du Canada, à Ottawa, en renferme de belles copies. On en trouve aussi quelquesunes aux archives de l'archevêché de Québec. Elles sont indiquées sous le titre "Documents du Vatican" (ibid., (1939-40): 155).

34 Carl Russell Fish, Guide to the Materials for American History in Roman and Other Italian Archives (Washington D.C., Carnegie Institution, 1911).

35 Ibid., iii.

36 Fish travailla principalement dans les archives romaines, mais il fit aussi des recherches à Naples, à Venise, à Turin et à Florence.

37 Ibid., 121-122.

38 Ibid., I.

39 Ibid. 
C'est pourquoi, si elle est aujourd'hui dépassée pour la partie qui porte sur les Etats-Unis au sens strict ${ }^{40}$, l'oeuvre de Fish reste fondamentale pour l'histoire du continent en général et pour celle du Canada en particulier.

Dans la section de son ouvrage où il traite de la Propagande, Fish adopte la démarche suivante: brève introduction historique, dans laquelle il fait état des problèmes de juridiction rencontrés par cette congrégation, et mentionne les fonds les plus importants et les noms de ceux qui y ont déjà travaillé en rapport avec l'histoire américaine ${ }^{41}$; suit la partie proprement documentaire: après une brève description de chaque série, Fish donne la liste des documents, notant en général le numéro du volume, le folio initial du document ou le numéro du dossier, et la date de la pièce, qu'il résume en une ou deux lignes, allant rarement jusqu'à trois. Fish signale tous les volumes examinés, même s'il n'y a rien trouvé. Il s'est penché sur les séries suivantes: Acta (pp. 123-147), SOCG (pp. 147158), Congressi (pp. 158-188), Udienze (p. 189), Cause (p. 189), Memoriali (pp. 189-190), Congregazioni particolari (p. 190-192), Miscellanea (p. 193-194), Scripta Varia (p. 195), Visite e Collegi (p. 195), Museo Etnografico (p. 195).

Toutefois, cet inventaire doit être consulté avec prudence. D'une part, les documents ont été en partie reclassés depuis l'époque de Fish: la numérotation des folios a été changée et certains volumes sont passés dans une autre série; d'autre part, Fish lui-même manque parfois de précision, fournissant pour certains documents le numéro du folio, pour d'autres le numéro du dossier, pour d'autres enfin seulement la date ou seulement le résumé, - sans parler des erreurs typographiques, néanmoins peu nombreuses vu la nature de l'ouvrage. Pressé par le temps, Fish s'est souvent contenté de lire les notes marginales (séries Acta et Congressi, par exemple) ou les résumés apparaissant au dos des documents (série $S O C G$ ), répétant les erreurs faites au cours des siècles par les archivistes de la Propagande, pas toujours bien renseignés ni capables de résumer correctement les documents.

40 Par le Calendar de Kenneally (voir note 53).

41 Il nomme, entre autres, Thomas J. Shahan, qui semble n'avoir connu que les bullaires qui concernent la Propagande et non pas les archives proprement dites (V. Thomas J. Shahan, "L'histoire de l'Église catholique aux États-Unis", Revue d'histoire ecclésiastique, I (1900): 678-689). 
D'autres erreurs sont imputables à Fish lui-même. Deux exemples illustreront à la fois sa façon de travailler et la source de certaines de ses méprises. Sous la rubrique Acta, on trouve: "Charles François Bailly de Mefrein deputed coadjutor"42. L'auteur eût-il connu le personnage en question, il aurait corrigé, entre parenthèses, Mefrein par Messein. Or la graphie Mefrein, évidemment due à un copiste distrait, n'apparaît que dans l'index du volume, le document même portant Messein. Fish n'a donc vu que l'index, sans lire le document. De même, encore sous la rubrique Acta, on trouve: "Leonard Neel (Neale) chosen coadjutor"43. Connaissant Neale, Fish a fait la correction. Or l'orthographe erronée ne se trouve que dans l'index et dans le rescriptum du document. Le document lui-même présentant la graphie correcte, il est évident que, cette fois encore, Fish ne l'a pas vu.

Les erreurs de Fish sont plus nombreuses dans la série $S O C G$, que l'on considère comme la plus difficile, et qu'on désignait autrefois sous le titre de Lettere Antiche ${ }^{44}$. Voici un échantillon de l'inventaire de Fish pour le volume 141 de la série $S O C G$, qui porte en général sur l'année 1642:

f. 26. May 14. The provincial of the Capuchins says, among other things, that the ambassador of Costantinople has been declared viceroy of North America.

f. 30. Jan. 20. The Capuchin missionaries of America designate a Capuchin priest as prefect of missions.

f. 31. May 28. The provincial of the Capuchins of France has received the decree for the extension of the missions in America.

f. 45. May 28, 1640. The provincial of the Capuchins of Normandy gives account of the missions of America.

ff. 51-52. May 26. The provincial of the Capuchins speaks of Canada and Florida, wishing to forestall the Dutch in occupying a place where the natives are docile and one can embark in February.

42 Voici la référence complète: “Acta, 1788, f. 316, no. 1, Sept. 10. Father Charles François Bailly de Mefrein deputed coadjutor of the Bishop of Quebec" (Fish, Guide, 141).

43 Voici la référence complète: “Acta, 1795. f. 8. Mar. 23. D. Leonard Neel (Neale) chosen coadjutor of the Bishop of Baltimore" (ibid.: 143).

44 La série SOCG (abréviation pour Scritture Originali Riferite nelle Congregazioni Generali) est divisée en deux parties. La première, connue sous le nom de Lettere Antiche, va du vol. 1 au vol. 417, et son matériel est rangé par aires géographiques ou par sujets. La deuxième correspond exactement aux Acta. 
f. 67. Nov. 7. The above mentioned provincial gives a relation of Canada.

ff. 68,73 . Dec. 8,1641 . Another relation of the same mission by the same provincial.

ff. 69-72. Oct. 8, 1641. The same provincial sends a map of parts of America, made by a priest who lived there three years.

ff. 216-218. Note of fourteen candidates considered for the mission of Maryland, with a relation of Maryland of Nov. 7, 1641 .

f. 222. Nov. 12, 1641. Decree of the Sacred Congregation on the expedition of missionaries to Maryland ${ }^{45}$.

Et voici le même échantillon, revu et corrigé46:

ff. 9 and 14. Jan. 24, 1642. The Capuchin provincial of Paris, Honoré de Cunières, gives an account of the missions of Greece, Canada, England. (Omis par Fish.)

ff. 10 and 13. Jan. 18, 1642. The Capuchin prefect of the mission of Canada, Pacifique de Provins, reports on Madagascar, Greece, Canada. (Omis par Fish.)

f. 24. Jan. 10, 1642. Pacifique writes of his friendship with Christopher of the Holy Trinity. The answer by Propaganda briefly touches on Canada. (Omis par Fish.)

ff. 30 and 37. Feb. 15, 1642. Pacifique is soon leaving for Canada. (Omis par Fish.)

ff. 38 and 41. March 7, 1642. Pacifique has received the decree for the extension of his mission to the whole of North América. (Omis par Fish.)

ff. 39-40 (Fish, f. 26). March 14(1642). The Capuchin prefect of the mission of Canada, Pacifique de Provins, says, among other things, that the French ambassador in Constantinople, Count de Marcheville, has been declared viceroy of North America.

f. 44 (Fish, f. 30). (Il s'agit seulement des Antilles.)

ff. 45-46 (Fish, f. 31). March 28 (1642). The Capuchin prefect of the mission of Canada, Pacifique de Provins, has received the decree for the extension of his mission to the whole of North America.

f. 68 (Fish, f. 45). (Il s'agit seulement des Antilles.)

45 Fish, Guide, 151.

46 Pour la révision de l'échantillon, je m'en suis naturellement tenu aux critères de Fish lui-même, sans m'arrêter à la qualité du résumé. J'ai ajouté les documents négligés par Fish, corrigé ses erreurs (les corrections sont en italique) et seulement mentionné les documents qui n'ont rien à voir avec l'Amérique du Nord. 
ff. $78-79$ (Fish, ff. 51-52). June 28, 1642. The Capuchin provincial of Paris, Honoré de Cunières, reports on the question of the Capuchin prefecture. Canada mentioned. (Omis par Fish.)

ff. 105 and 116 (Fish, f. 67). Nov. 7, 1641. The above mentioned prefect gives a relation of Canada.

ff. 106 and 115. Feb. 4, 1642. A note by Propaganda on the extension of Pacifique's mission. (Omis par Fish.)

ff. 107 and 114 (Fish, ff. 68, 73). Dec. 12, 1641. Another relation on Canada by the same prefect.

ff. 108 and 113 (Fish, ff. 69-72). Oct. 17, 1641. The same prefect sends a map of New France, made by a priest who lived there three years.

ff. 122 and 127. Dec. 30, 1641. Christopher of the Holy Trinity reports on New France and Pacifique's projects. (Omis par Fish.)

ff. 346-351 (Fish, ff. 216-218). Various items. Letter of Carlo Rossetti, Pope's minister to England, to Propaganda, of Sep. 7, 1641. Note of fourteen candidates considered for the mission of Maryland. A relation on Maryland, undated. Notes by Propaganda on the whole dossier.

ff. 356 and 363 (Fish, f. 222). Nov. 12 (1641). Decree of the Sacred Congregation on the expedition of missionaries to Maryland.

Les erreurs, distractions et omissions ne manquent donc pas. Néanmoins, utilisé avec prudence, l'inventaire de Fish reste encore aujourd'hui indispensable.

Au contraire, on cherchera en vain l'utilité - par rapport toujours à la Propagande - de l'imposante thèse de doctorat d'Achille Gingras ("Guy Laviolette") ${ }^{47}$, qui devrait répertorier tout le matériel d'intérêt canadien conservé dans les archives de Paris, de Rome et de Londres. La partie qui concerne la Propagande (pp. 238-323) s'ouvre sur une brève histoire de cette congrégation et sur un aperçu sommaire de ses différents fonds. Rien là de nouveau par rapport à Fish. D'emblée, Gingras commet au surplus deux graves erreurs: il confond la série $S O C G$ avec la série Congressi ${ }^{48}$ (erreur d'autant plus grave qu'il s'agit de deux séries fondamentales et

47 Achille Gingras (Guy Laviolette), Répertoire des sources manuscrites de l'histoire religieuses canadienne en Europe surtout à Paris, à Rome et à Londres: 1608 à 1860, thèse, Institut Catholique de Paris (1958-59).

48 Ibid., 240. 
plutôt volumineuses), et il attribue 417 volumes $^{49}$ (à l'instar de Fish $^{50}$ ) à la série Memoriali, laquelle fait aujourd'hui partie de $S O C G$ et ne compte par elle-même que 36 volumes (de 382 à 417 ). Ces deux erreurs se répercutent évidemment dans la liste des documents établie par Gingras.

Cette liste de documents (pp.242-323) diffère de celle de Fish, lequel avait adopté une démarche archivistique, énumérant les volumes dans leur ordre topographique, pour ensuite en décrire le contenu, - en ceci que Gingras suit un ordre chronologique. Il en arrive à des regroupements, plus ou moins homogènes quant aux sujets traités, de références comportant en principe la date du document et la mention de la série à laquelle il appartient. Voici un exemple:

Relation sur les provinces et couvents des Franciscains, adressée au Cardinal Barberini, leur protecteur.

Mémoire à l'usage des nonces de France pour empêcher les hérétiques de pénétrer au Canada. (19 janvier 1635).

État des missions du Canada.

Scritt. Rif., vol. 259, ff. 30-60; 109 à 115.51

Le procédé n'est pas des plus clairs. Et encore Gingras n'est-il pas constant, négligeant parfois la date, parfois le numéro du folio ou du dossier, et quelquefois même la mention des séries. Or tout cela n'est pas le fruit du hasard, Gingras s'étant limité à copier la liste de Fish, délestée de tout le matériel qui lui parut (exclusivement sur la base des sommaires de Fish) sans intérêt pour le Canada. À l'examen, on se rend compte, en effet, qu'il est nécessaire d'avoir sous les yeux la liste de Fish pour utiliser celle de Gingras; en outre, Gingras cite l'ancienne numérotation des folios, en usage à l'époque de Fish mais depuis longtemps remplacée en 1958; enfin, Gingras ne cite aucun document qui ne soit déjà dans Fish. Tout au plus Gingras ajoute-t-il les siennes aux erreurs de Fish: incompréhension

49 Ibid.

50 Fish, Guide, 189.

51 Gingras, Répertoire, 242. 
ou fautes de frappe, entre autres, - ce qui se produit vingt et une fois pour la période de 1622 à 179952 . En ce qui concerne la Propagande, l'inventaire de Gingras est donc parfaitement inutile.

L'inventaire le plus important qui ait jamais été publié des archives de la Propagande relatives aux États-Unis est le Calendar qui, sur l'initiative de l'Academy of American Franciscan History, a été compilé par Anton Debevec et édité par Finbar Kenneally. Sept volumes ont paru à ce jour ${ }^{33}$; d'autres sont en préparation. Commencé par Debevec dès 1955, le dépouillement des archives a été mené jusqu'à l'année 1863 pour la First Series; la Second Series couvrira tous les documents de la période 1864-1908, dont le dépouillement est déjà avancés4.

52 Voici la liste des erreurs qu'on peut faire remonter directement à Fish:

p. 241, ligne 14: "17 nov." au lieu de "11 nov.".

p. 242, ligne 12: "Le P.V." au lieu de "Le P. Hyacinthe".

p. 242, ligne 34: "ff. (...) 359" au lieu de "ff. (...) 269".

p. 243, ligne 21: "trois Capucins" au lieu de "quatre Capucins".

p. 243, ligne 29: "f. 36" au lieu de "f. 367".

p. 244, ligne 37 : “13 mars 1645" au lieu de "13 mars 1646".

p. 244, ligne 39: " 27 avril 1645" au lieu de " 27 avril 1646".

8 p. 244 , ligne 43 : "25 octobre 1645" au lieu de " 20 septembre 1645 et 25 octobre 1646".

9 p. 245 , ligne 22 : “10 déc. 1647" au lieu de "17 déc. 1647 ".

10 p. 245, ligne 29: "lettre du nonce" au lieu de "lettre de Pacifique de Provins".

11 p. 247 , ligne 1: "27 mai 1659" au lieu de "27 mai 1661".

12 p. 247 , ligne 7: " 13 juin 1660 " au lieu de " 13 janv. 1660 ".

13 p. 247 , ligne 31 : “4 fév. 1664" au lieu de " 30 juin 1664" ("4 fév. 1664 " est la date de la rubrique précédente dans la liste de Fish).

14 p. 249, ligne 10: "No. 28" au lieu de "No. 25".

15 p. 250 , ligne $2:$ " 4 mars" au lieu de "4 février".

16 p. 250, ligne 32: "Récollets de Saint-Domingue" au lieu de "Récollets de Saint-Denis".

17 p. 255, ligne 20: “3 mars 1765" au lieu de "3 mars 1766" (l'erreur vient ici d'une distraction de Fish, qui attribue au volume des Acta l'année 1765, alors qu'il s'agit évidemment de l'année 1766).

18 p. 255 , ligne 23 : "f. 96 " au lieu de "ff. 93-96".

19 p. 258 , ligne 21 : "fol. 115 " au lieu de "fol. 114 ".

20 p. 260 , ligne 3 : "fol. 55 " au lieu de "ff. 50-75".

21 p. 260 , ligne 30 : "fol. 224" au lieu de "ff. 223-225".

53 Finbar Kenneally, ed., United States Documents in the Propaganda Fide Archives. A Calendar. First Series (Washington,D.C., Academy of American Franciscan History (7 vol.), 1966-1977). J'ai déjà écrit deux comptes rendus de cette oeuvre, dont l'un est très détaillé (Rivista Storica Italiana, LXXXVII, 3 (settembre 1976): 599-605), l'autre plus général (The William and Mary Quarterly, 3rd ser., XXXV, 2 (April, 1978): 419-420).

54 On voudra bien tenir compte de ce qui a été dit à la note 6 . Les microfilms des documents analysés dans le Calendar sont déposés à la Notre Dame University (V. note 16). 
Le premier volume est préfacé par Kenneally, qui explique le but poursuivi et le travail en cours. Puis, dans une introduction, Antonine Tibesar fait l'histoire de la Propagande, décrit les différentes séries qui constituent ses archives et signale les plus grandes difficultés rencontrées pendant la préparation du Calendar. (Aucun autre volume ne comporte d'introduction.) Viennent ensuite les "fiches" documentaires proprement dites, sur deux colonnes. Chaque "fiche" est précédée d'un numéro d'ordre; suivent la numérotation des folios et du volume, le titre du document ou, dans le cas d'une lettre, le nom de l'auteur et du destinataire, la date, le lieu d'origine, un sommaire (concis mais complet) du contenu, une liste des noms mentionnés et, finalement, l'indication de la langue dans laquelle est rédigé le document. Debevec a trouvé, lu, traduit et ordonné tous ces documents; il en a préparé les sommaires. Il a accompli une tâche gigantesque, dont le résultat est un ensemble de "fiches" extrêmement soignées et précises, auxquelles on peut se fier entièrement. Son oeuvre est de celles qu'il est permis de dire définitives.

On peut, cependant, déplorer deux choses, au sujet de ce Calendar. D'abord, que l'editing n'en soit pas plus rigoureux: index inutiles, sinon trompeurs et nuisibles; séries morcelées, éparpillées dans plusieurs volumes ou plusieurs parties d'un même volume; index analytiques inadéquats, restreints aux seuls noms propres et comportant beaucoup d'erreurs; enfin, fautes de typographie. Tout cela nécessitera la publication d'un volume entièrement consacré aux index, à la fin de la First Series. On peut aussi regretter l'orientation même du projet, trop restreint dans ses limites territoriales. Dans les archives de la Propagande, par "America Settentrionale" on entendait le Canada actuel; par "America Centrale", le territoire qui s'étend du Canada à Panama; par "America Meridionale", l'Amérique du Sud. En limitant leur recherche aux seuls territoires des Etats-Unis actuels, on a écarté du même coup tout le territoire canadien. Il ne s'agit pas tant, ici, du travail déjà fait ${ }^{55}$ quasi en pure perte pour le Canada, que d'une

s5 Au cours de ses recherches, Debevec a consulté attentivement tout ce qui concerne le Canada. À preuve l'inventaire dactylographié déposé au Centre de Recherche en Histoire religieuse du Canada de l'Université Saint-Paul, dont le titre est Prop. Fide Archives, préparé par Debevec lui-même, et qui contient des "fiches" d'intérêt canadien pour les séries $A$ cta (vol. 3-17), SOCG (vol. 74, 100-102, 105, 129, 131-133, 135, 138, 259, 347), Congressi America Settentrionale (vol. 1), Lettere (vol. 4-13, 15). Il est évident que bien des "fiches" du Calendar concernent non seulement les Etats-Unis, mais aussi le Canada. 
conception méthodologique erronée concernant l'Amérique du Nord et, en particulier, la pénétration catholique sur le continent au $\mathrm{XVIIe}$ et au XVIIIe siècle, à une époque où les frontières étaient extrêmement imprécises entre ces deux pays dont l'histoire s'entremêlait continuellement. Malgré tout, le Calendar reste une oeuvre de première importance et qui, pour les États-Unis, dépasse nettement l'inventaire de Fish.

\section{Documents de la Propagande en Amérique du Nord}

La question des copies de documents provenant de la Propagande qui sont conservées dans les dépôts d'archives et les bibliothèques de l'Amérique du Nord est assez complexe et n'est qu'à demi éclaircie. Aux États-Unis, c'est la Georgetown University qui conserve les copies manuscrites que fit faire autrefois John Dawson Gilmary Shea ${ }^{56}$, et la Notre Dame University qui conserve les microfilms des documents inventoriés dans le Calendar édité par Kenneally, tandis que les copies qui servirent à l'édition des Carroll Papers $^{57}$ sont déposées à la Catholic University of America.

Le cas du Canada est différent. À ce qu'on disait autrefois ${ }^{58}$, il devrait exister des copies de documents de la Propagande aux Archives de l'Archevêché de Québec et aux Archives publiques du Canada. Une visite (trop rapide) aux Archives de l'Archevêché de Québec n'a pas permis, malgré l'aide et le dévouement de l'archiviste Armand Gagné, d'y découvrir copie d'un seul document de la Propagande. Il s'y trouve, cependant, une liste de documents, à l'usage d'un copiste qui travaillait à Rome, qui correspond exactement aux documents qui sont aujourd'hui copiés dans un cahier daté de 1884 et conservé aux Archives publiques du Canada ${ }^{59}$.

56 Selon E. I. Devitt, ces copies sont conservées à la Georgetown University: "Dr Shea's MSS. in Georgetown University Archives contain a voluminous collection entitled 'Propaganda Transcripts', which include nearly all of these documents, many of which were translated and published in full by him" (E. I. Devitt, ed., "Propaganda Documents. Appointment of the First Bishop of Baltimore", American Catholic Historical Society of Philadelphia, Records, XXI, 4 (December, 1910): 186). Je n'ai pas encore eu la possibilité de consulter personnellement ce fonds.

57 Thomas O'Brien Hanley, ed., The John Carroll Papers (3 vol.) (Notre Dame, Ind., University of Notre Dame Press, 1976).

58 Morin, "Archives du Saint-Siège": 170-176; Caron, "Inventaire": 155: "Documents relatifs au Canada": 106. V. aussi Caron, "Les archives de l'Archevêché de Québec", $L a$ Société Canadienne d'Histoire de l'Église Catholique, Rapport (1934-35): 72.

sy Il s'agit de quatre documents indiqués comme Mis., I-I49a, Mis., I-I49b, Mis., I149c, Mis., III, N. 17 . 
Les copies de documents de la Propagande conservées par les Archives publiques du Canada sont décrites avec une suffisante précision dans l'Inventaire général60, en particulier, sous la cote MG 17 (Archives religieuses), A (Église catholique) 1 (Vatican: Archives secrètes du Vatican), et sous le titre "Sacrée Congrégation de la Propagande", on trouve trois volumes, qu'on peut ainsi identifier:

Vol. 1 (a): "Correspondance des évêques". Il s'agit de 443 pages manuscrites tirées de la Propagande, série SOCG, volumes 256, 259, 260, et série Congressi America Settentrionale, volume 1. Le tout est relié sous la forme d'un cahier, daté de 1884, qui indique précisément comment ces copies ont été faites pour l'archevêque de Québec, Elzéar-Alexandre Taschereau, par l'intermédiaire de l'abbé Henri-Raymond Gasgrain. Ce cahier correspond à la liste des Archives de l'Archevêché de Québec dont il a été question précédemment.

Vol. I (b): "Ettat de la mission d'Acadie, 1656". C"est le compte rendu, "Brevis ac dilucida" ("court et clair"), d'Ignace de Paris, avec traductions en anglais et en français, et des notes de Candide de Nant. Le document provient de la série $S O C G$, volume 260 , ff. 38rv - 48rv.

Vol. I (c): "Lettre de l'évêque de Pétrée au pape". Cette lettre du vicaire apostolique François de Laval au pape ne provient pas de la Propagande, mais des Àrchives secrètes du Vatican. Vol. 1 (d): "Extraits, 1630-1646". Cahier de 23 pages, qui concerne plutôt les années 1630-1650 et qui contient des documents de la Propagande, série Acta (plusieurs volumes) et série $S O C G$, volume 144 .

Vol. 2: "actes ou extraits". Ces 427 pages d'extraits ou de copies intégrales de documents de la Propagande proviennent de la série Acta (plusieurs volumes, de l'année 1625 à l'année 1820). Vol. 3: On y trouve la traduction française du volume 2, précédée d'un index du volume 3.

Ces copies, que conservent les Archives publiques du Canada, sont le résultat d'initiatives diverses et qui remontent parfois assez loin dans le temps. D'aucune manière elles ne forment un ensemble organisé, et leur intérêt diminuera notablement quand les projets parallèles des Archives publiques du Canada et de l'Université Saint-Paul, du côté canadien, et de l'Academy of American

60 Archives publiques du Canada, Division des Manuscrits, Inventaire général. Manuscrits, III, MG I7-MG 21 (Ottawa, 1974): 3 . 
Franciscan History, du côté américain, auront été menés à bien ${ }^{61}$. À ce moment-là, tout le matériel documentaire concernant l'histoire du Canada et des États-Unis que recèlent les archives de la Propagande aura été inventorié, et, grâce au microfilm, on pourra le consulter sans avoir à quitter l'Amérique du Nord.

Peut-être, à ce moment-là aussi, sera-t-il nécessaire d'étendre le projet aux autres archives religieuses de Rome.

61 Pour ce qui est du projet canadien, l'inventaire a commencé en octobre 1977; pour le moment on examine la période 1622-1799. L'inventaire de la série Acta est déjà disponible, ainsi que les microfilms correspondants; l'inventaire de la série $S O C G$ est en préparation. Quant au projet américain, l'inventaire de la période postérieure à 1864 est déjà avancé. 